\title{
The Role of Cardiovascular Magnetic Resonance in Adults with Congenital Heart Disease
}

\author{
Philip J. Kilner ${ }^{*}$ \\ CMR Unit, Royal Brompton Hospital and Imperial College, London, UK
}

\begin{abstract}
The comprehensive coverage and versatility of cardiovascular magnetic resonance (CMR), providing functional as well as anatomical information, make it an important facility in a center specializing in the care of adults with congenital heart disease. Imaging specialists using CMR to investigate acquired heart disease should also be able to recognize and evaluate previously unsuspected congenital malformations. Conditions that may present or be picked up during imaging in adulthood include atrial septal defect, anomalously connected pulmonary veins, double-chambered right ventricle, congenitally corrected transposition of the great arteries, aortic coarctation, and patent arterial duct. To realize its full potential and to avoid pitfalls, CMR of adults with congenital heart disease requires specific training and experience. Appropriate pathophysiological understanding is needed to evaluate cardiovascular function after surgery for tetralogy of Fallot, after transposition of the great arteries, and after Fontan operations. For these and other more complex cases, CMR should ideally be undertaken by specialists committed to long-term collaboration with the clinicians and surgeons managing the patients in a tertiary referral center.
\end{abstract}

\section{Keywords}

Adult congenital heart disease; MRI; Imaging; Tetralogy of Fallot; Transposition of the great arteries; Atrial septal defect; Ventricular septal defect; Aortic coarctation

Congenital heart disease (CHD) occurs in about 6 to 8 of 1000 live births. ${ }^{1,2}$ Without intervention, the prognosis for more complex forms is poor. However, advances in pediatric cardiology and cardiac surgery over the last few decades have revolutionized patient management; and most patients now survive into adulthood. This has led to new challenges as increasing numbers of congenital heart patients pass into the care of adult cardiac services. The need for expert knowledge to appropriately investigate the highly variable anatomy and pathophysiology and to manage this patient group has led to the expanding cardiological subspecialty of adult congenital heart disease (ACHD). ${ }^{3,4}$ Many ACHD patients will have undergone palliative or reparative surgery during early life. These procedures are rarely curative, and lifelong follow-up is generally required to optimize the quality and span of life.

More than one imaging modality is likely to be needed to address all the relevant clinical questions, particularly in the more complex ACHD cases. Although transthoracic

(C) 2011 Published by Elsevier Inc.

*Address reprint requests to CMR Unit, Royal Brompton Hospital and Imperial College, SW3 6NP London, UK. p.kilner@rbht.nhs.uk. .

Statement of Conflict of Interest The author declares that there are no conflicts of interest. 
echocardiography is the first-line cardiovascular imaging modality, acoustic windows are rarely adequate for access to all regions of interest. The choice of further imaging depends on the clinical questions that remain to be addressed. Transesophageal echocardiography gives excellent access to more posterior parts of the heart, particularly for 3-dimensional (3D) visualizations of the valves, septal defects, and infective vegetations, and is suitable for intraoperative use. The disadvantages of transesophageal echocardiography include its relatively invasive nature, generally requiring sedation or anesthesia, and its limited fields of penetration. Computed tomography (CT) has the advantage of providing excellent, unrestricted spatial resolution in a short acquisition period, ${ }^{5}$ but with less versatility than CMR and with a radiation dose that may be of concern in relatively young patients who could require repeated investigations over time. ${ }^{6}$ However, CT is likely to be preferred in a few specific situations, for example, in patients with a permanent pace-maker, for the investigation of stent lumen and possible aneurysm formation after balloon dilatation and stenting of aortic coarctation, or where small vessels such as the coronary arteries ${ }^{7}$ or aortopulmonary collaterals need to be visualized clearly. Cardiac catheterization is used less often for diagnosis in ACHD since improved noninvasive approaches have became available; but catheterization still has roles in calculations of pulmonary resistance, in assessments of coronary artery disease, and for percutaneous interventions using balloons, stents, occlusion devices, or implantable valves, notably for placement of a valve in preexisting but regurgitant right ventricle (RV) to pulmonary artery (PA) conduit.

Many ACHD patients develop arrhythmias secondary to intrinsic conduction abnormalities, surgical interventions, or residual hemodynamic lesions. It is important for imaging specialists to understand the palliative shunts, surgical repairs, and reconstructive procedures used, some of which entail surgical procedures that have changed greatly over the years. ${ }^{8,9}$ For those ACHD patients known to be liable to need further arrhythmic, hemodynamic, or surgical intervention, it is recommended that CMR is undertaken in the specialist center where they are being followed up..$^{10}$ Examples include patients with restenosis or aneurysm formation after repair of aortic coarctation, those with pulmonary regurgitation (PR) after repair of tetralogy of Fallot (ToF), those who have undergone surgery for transposition of the great arteries (TGA) or for a functionally single ventricle, and a variety of other more unusual or complex conditions. The variety of underlying and postoperative conditions encountered combined with the versatility of CMR makes for a great deal of choice regarding how to image a particular patient. Further information on this is available in the European Society of Cardiology recommendations for CMR of ACHD. ${ }^{10}$ Not only is relevant experience needed to acquire and interpret images in more complex ACHD patients; but collaboration between the imaging specialists, cardiologists, and surgeons in such centers contributes to improved understanding of the complex and variable conditions that they have to deal with. Large imaging data sets with acquisitions of various types are best reviewed interactively in the context of multidisciplinary discussion of clinical management.

General radiologists and cardiac imaging specialists also need to be able to recognize and appropriately investigate previously undiagnosed CHD that may present in adulthood and at least appreciate the nature and potential complications of the main types of CHD. Besides aortic coarctation as a possible cause of upper limb hypertension, clinicians should be alert to atrial septal defect (ASD) as a possible cause of a dilated RV, including sinus venosus defects, which may not be directly visible on routine echocardiography and are commonly associated with partial anomalous pulmonary venous drainage. Ventricular septal defect (VSD), double-chambered RV (subinfundibular stenosis), and patent ductus arteriosus (PDA) can all present in adulthood, possibly as an audible murmur or through the dilatation of a chamber or vessels seen on a chest radiograph. Congenitally corrected transposition (atrioventricular and ventriculoarterial discordance) can also remain undiagnosed until 
adulthood, and it is important to be able to distinguish a morphologically right from a left ventricle $(\mathrm{LV})$ from cross-sectional imaging.

\section{Approaches to comprehensive anatomical coverage}

Except in straightforward cases such as aortic coarctation where the questions to be answered are well defined, it is prudent in a baseline or presurgical CMR study of ACHD to perform a comprehensive examination that will allow review of the structure and, if possible, the function of the myocardium, valves, and vessels through all regions of the heart and mediastinum. There are several possible approaches to this; and a choice should be made, balancing time taken against information gained.

Firstly, it takes little time ( 2 or 3 additional breathholds) to acquire stacks of static multislice images in coronal and sagittal as well as transaxial orientations in all cases. Bright-blood, balanced steady-state free precession (SSFP) acquisitions have the advantage of good, rapid acquisition, for example, as a slice per heartbeat. They give good visualization of the pulmonary veins and other vessels and may show up jets that are flowing at the time of acquisition.

In complex or unpredictable cases, my preferred approach to achieving comprehensive coverage of structures and movements, including information of jet flow, is the acquisition of a contiguous stack of SSFP cine images; typically 5-mm slice thickness, without gaps and in the transaxial orientation. Such a transaxial cine stack is easy to review without postprocessing, enabling a search up and down through the mediastinum to assess cardiovascular connections, myocardial movements, and vascular wall displacements. The cines also show evidence of any jet formation at a stenosis, valve leak, or shunt. Two or possibly 3 slices may be acquired per breath-hold, depending on a patient's respiratory ability. Coverage can be from the diaphragm to the aortic arch, unless a more limited region is in question. Cine stacks in oblique orientations should also be considered, particularly orientated perpendicular to the thin, extended structures of regurgitant mitral valve leaflets or to the atrial septum in the presence of a defect.

Dynamic contrast-enhanced angiography or noncontrast, cardiac-gated 3D SSFP acquisition can also be valuable, although these methods do not, on their own, yield cyclic functional information. They can be post-processed to provide 3D images or reformatted in planes of any orientation.

A comprehensive approach to flow imaging is 3D, 3-directionally encoded and timeresolved velocity acquisition by phase contrast CMR, widely known as $4 D$ velocity mapping. ${ }^{11}$ It currently entails acquisition periods of 10 minutes or more and needs relatively specialized postprocessing. It depicts relatively large-scale, multi-directional flow features, but is not yet convincingly suited to routine clinical investigation. Shorter acquisition times and appropriate postprocessing tools would be needed for it to become an attractive clinical option, for example, for the quantification of flow in several vascular regions in the presence of regurgitant and/or shunt lesions.

\section{Measurement of right ventricular volumes}

Functional assessment of the right heart represents an important aspect of imaging in ACHD patients. ${ }^{12}$ Several aspects of the RV present challenges for reproducible volumetric analysis by CMR. Several different types of software are available for such measurements; and unfortunately, there is not yet a consensus as to which is preferable. Whichever is used, it is important to maintain consistent methods of acquisition and segmentation with a view to longitudinal comparison between studies. The free wall and apical regions of the RV are 
normally extensively trabeculated, with relatively little compact myocardium; and the trabeculations become more apparent with hypertrophy. A decision has to be made whether to include or exclude trabeculations from the blood volume. Smooth tracing outside the trabeculations and inside the compact layer is probably the most reproducible, but not necessarily the most accurate, approach in the presence of RV hypertrophy. The long-axis displacement of the base of the RV is usually greater than that of the LV, and it is important to include this basal region when locating the first short-axis slice at end diastole. After repair of ToF, any thin-walled, akinetic region of the RV outflow tract should be included in $\mathrm{RV}$ volume, up to the level of the pulmonary valve annulus.

\section{Some specific categories of CHD}

\section{Shunts: ASD, VSD, or PDA}

Each of these may present in adulthood or be a residual lesion in a previously operated patient. Echocardiography is usually the modality of choice for identifying or excluding septal defects; and in the case of a suspected but uncertain patent foramen ovale (PFO), repeated echocardiography with contrast is likely to be more informative than CMR. However, for unusual septal defects, CMR can add information, ${ }^{13,14}$ including shunt quantification and measurement of biventricular size and function.

Four types of ASD can be distinguished; and each can be visualized by appropriately orientated stacks of CMR cine images, no more than $5 \mathrm{~mm}$ thick and without gaps, followed by velocity mapping. For the most common, ostium secundum ASDs, an atrial short-axis stack of cines is recommended, orientated as for the routine ventricular short-axis stack, but stepping back from the atrioventricular junction through the atria until the superior vena cava (SVC) is seen. A secundum ASD is illustrated in (Fig 1), in the setting of Ebstein anomaly. For a superior sinus venosus defect, a transaxial cine stack is recommended, stepping up through the atrial cavities to the SVC, where the commonly associated anomalous right upper pulmonary vein(s) draining to the SVC may be found. ${ }^{15}$ If a superior sinus venosus defect is found, an oblique cine in a 3-chamber ( $\mathrm{LV}$ outflow tract) orientation and passing through the defect offers an additional view. For an inferior sinus venosus defect, an oblique sagittal cine stack, orientated perpendicular to the lower part of the atrial septum, gives views of the defect in relation to the inferior vena cava and inferior wall of the left atrium. Finally, for an atrioventricular septal defect, a stack of cines in the 4-chamber orientation shows the $\operatorname{defect}(\mathrm{s})$ in relation to the atrioventricular valve leaflets and ventricular septum.

Through-plane velocity mapping, velocity encoding range set at $100 \mathrm{~cm} / \mathrm{s}$ and orientated orthogonal to the flow through the ASD, en face to the septum, allows delineation and sizing of the defect(s).

For recognizing VSDs, a systolic jet into the RV in one of the routine short-axis cines, usually basal, is a common clue on CMR, to be followed up by further cross-cut or stacked cines and through-plane velocity mapping.

A PDA may be suspected on multislice scout images, particularly if acquired by SSFP, but needs confirmation by aligning a cine with the jet flow and transecting the jet with a velocity map.

For shunt measurement, ascending aortic flow measured by CMR represents systemic flow (systemic flow volume) when there is an intracardiac left to right shunt. However, when the shunt is from the descending aortic arch through a PDA, it is the ascending aortic flow that is increased and is approximately equivalent to the amount of flow distal to the duct, into the 
pulmonary vessels (pulmonary flow volume). The smaller amount of flow measured in the pulmonary trunk will be equivalent to the systemic flow volume. ${ }^{16}$

\section{Aortic coarctation}

Although most surgical repairs and catheter interventions for aortic coarctation have satisfactory outcomes, a mild degree of residual aortic narrowing is common; and it may be important to screen periodically for restenosis ${ }^{17}$ or aneurysm formation. Aneurysms may occur after attempted balloon dilatation or after repairs by a prosthetic patch, which is rarely if ever used now. Cine images aligned with the arch and, importantly, with the jet passing through the narrowest region, followed by the mapping of jet velocity through a plane aligned to transect the jet immediately distal to the stenosis, provide adequate follow-up assessment in most cases. However, contrast-enhanced angiography should be acquired in any case likely to require catheter intervention or surgery, either for restenosis or for aneurysm formation.

Echocardiography from a suprasternal window can usually provide a Doppler trace of jet flow through the residual coarctation, enabling an assessment of the presence or absence of diastolic prolongation of forward flow ("diastolic tail"), which can be a telling sign of significant obstruction. However, 2-dimensional echo rarely provides full visualization of the repaired region in adults. Because repeated imaging is likely to be needed through the course of a lifetime, CMR is probably the modality of choice for assessment every few years or sooner if there is specific cause for concern. ${ }^{18}$ An exception is after deployment of a stent, when at least one contrast CT study provides better assessment of the instent lumen and any possible aneurysm formation in the vicinity of the stent. ${ }^{19}$

A functionally bicuspid aortic valve, present in at least $50 \%$ of coarctation cases, requires assessment because it will be more prone to progressive stenosis and/or regurgitation than a normal valve. Berry aneurysms of the circle of Willis or other cerebral vessels occur in up to $10 \%$ of patients with coarctation bearing the risk of rupture, ${ }^{20}$ and screening for cerebrovascular aneurysms by cerebral magnetic resonance angiography may be considered appropriate.

\section{Marfan syndrome}

As measurement of the aortic root, ascending aorta, its branches, and any aortic regurgitation is likely to be needed periodically, CMR is likely to be the modality of choice. It is important to check for aortic dissection, which may be small and focal. ${ }^{21}$ The LV and mitral valve should also be assessed. Magnetic resonance imaging of the lumbosacral spine may be used to identify dural ectasia. ${ }^{22}$

\section{Valve disease}

Although echocardiography remains the first line of investigation, ${ }^{23,24} \mathrm{CMR}$ can contribute to the measurement of regurgitation, the visualization of jets in relation to valve leaflets, and the assessment of myocardial or other pathology associated with heart valve disease. In patients with a bicuspid aortic valve, CMR allows assessment of any ascending aortic ectasia, aneurysm, or dissection. Regurgitant or stenotic jets are visible on cine imaging, although appearances depend on the jet size and characteristics and on the relative location and orientation of the imaging slice. The visible extent of a jet does not necessarily relate to the severity of a lesion. Planimetry of an orifice, or of the cross section of the jet immediately downstream of the orifice, is feasible in some but not all cases by cine CMR, depending on the structure of the jet and the relative thickness and location of the imaging slice. A stack of 4-mm-thick SSFP cine slices perpendicular to the jet close to its origin is recommended. Cardiovascular magnetic resonance jet velocity mapping can contribute to 
quantification of stenosis, but velocities may be under-estimated if the slice is not optimally located or if the coherent core of the jet is too narrow or fragmented to contain several whole voxels. An appropriately aligned and encoded in-plane jet velocity acquisition may be helpful to locate the region of highest velocity before through-plane velocity measurement. For each of these, it is important to bear in mind voxel dimensions, particularly slice thickness, relative to the shape and orientation of a jet and the potential for velocity underestimation due to partial volume averaging. Quantification of regurgitation of the inflow valves is feasible by CMR using ventricular stroke volume difference in the case of a single valve lesion or by subtraction of the outflow volume, measured by mapping velocities through a plane transecting the relevant great artery, from the corresponding ventricular stroke volume. For the identification of tethering, prolapse, or failure of coaptation of all parts of the mitral (or tricuspid) leaflets, a contiguous stack of cine images aligned perpendicular to the central part of the line of coaptation is recommended. ${ }^{25,26}$

\section{Ebstein anomaly and tricuspid regurgitation}

In Ebstein anomaly, the septal leaflet, particularly its more inferior parts, is apically displaced with "atrialization" of the basal part of the RV. In some severe cases, the tricuspid valve plane can be rotated to lie in an almost horizontal plane beneath a dilated RV outflow tract. Such malformation is well visualized by CMR, using stacks of transaxial and shortaxis cines and 4-chamber and an oblique coronal (or RV vertical long-axis) cine to show the displacement of the inferior insertion of the tricuspid valve (Fig 1). The severity of tricuspid regurgitation (TR) can be assessed using through-plane CMR velocity mapping, the velocity encoding range typically set at $250 \mathrm{~cm} / \mathrm{s}$, to depict the cross section of the regurgitant stream through a plane transecting the jet immediately on the atrial side of the defect. A regurgitant jet cross section, reflecting the regurgitant defect, of $6 \times 6 \mathrm{~mm}$ or more can be regarded as severe. An ASD, due to distension and gaping of a patent foramen ovale, can be present in about 50\% of adult Ebstein patients and should be sought by echo or by a CMR atrial shortaxis cine stack.

Repaired ToF-Important issues in the relatively large subgroup of patients who have undergone surgical repair of ToF are the size and regional as well as global function of the $\mathrm{RV}$, the function of the pulmonary valve, and the patency of the right and left PAs. ${ }^{27}$ Free or almost free PR is common. Dysfunction of the LV, aortic dilatation, TR, and a residual VSD patch leak are less common problems. Echocardiography can usually address most but rarely all of these, which gives an important role to CMR. ${ }^{28,29}$ The pathophysiology of PR differs from that of aortic regurgitation. Free PR, with little or no effective valve function, may be tolerated without symptoms for decades and is typically associated with a regurgitant fraction of approximately $35 \%$ to $45 \% .{ }^{30} \mathrm{It}$ is often associated with a triphasic pattern of flow in the pulmonary trunk-systolic forward flow, early to mid diastolic reversal of flow (regurgitation), and then late diastolic forward flow as atrial contraction boosts forward flow through a fully expanded and therefore conduit-like RV (Fig 2). Right ventricle dysfunction, arrhythmia, and premature death can result. In most centers, pulmonary valve replacement is considered in such patients; but when to operate remains controversial, particularly if the patient is asymptomatic and bearing in mind that a homograft replacement may only function effectively for 15 or 20 years, or less. ${ }^{31-33}$ Once a conduit is in position, however, progressive stenosis or regurgitation may be treatable by percutaneous placement of a stented valve within the relatively rigid tube of the conduit. ${ }^{34,35}$ Cardiovascular magnetic resonance, with contrast angiography, has a role in the selection of patients for such procedures; but so also has CT, which llows the visualization of calcium and the clear delineation of the coronary arteries relative to a previously placed conduit. 
Even in the absence of an effective pulmonary valve, the amount of regurgitation depends on factors upstream and downstream. In occasional cases, the regurgitant fraction can exceed 50\%. This may be attributable to an unusually large and compliant RV, a large and compliant pulmonary trunk, and PA branches whose recoil contributes to the regurgitation, ${ }^{36}$ branch PA stenosis, or elevated peripheral resistance limiting the distal escape of flow, or combinations of these. ${ }^{37}$ In summary, the evaluation of repaired ToF requires thorough assessment of the left and right heart, extending to the branch PAs and ascending aorta.

\section{Double-chambered RV or subinfundibular stenosis}

This condition results from obstructing muscular bands or ridges between the hypertrophied body or sinus of the RV and the nonhypertrophied infundibulum. It is usually associated with a VSD into the higher pressure part of the RV, close to the upper part of the tricuspid valve, and may progress during adulthood. The subinfundibular origin of the jet in doublechambered RV, directed into the nonhypertrophied and nonobstructive infundibulum, is generally visible in routine basal short-axis cines (Fig 3). ${ }^{38}$

\section{Major aortopulmonary collateral arteries}

Contrast-enhanced 3D CMR angiography may be used for delineation of the sources of pulmonary blood supply before surgical or transcatheter procedures in patients with major aortopulmonary collateral arteries that generally occur secondary to pulmonary atresia or severe stenosis. ${ }^{39}$ However, CT angiography is likely to provide better resolution and may be preferable.

\section{Pulmonary hypertension}

Doppler echocardiography has the important advantage of being able to measure the velocities, and hence the presumed intercavity pressure difference, of relatively narrow jets of TR or PR. This cannot be done reliably by CMR. However, CMR does give good visualization of RV size, function, hypertrophy, any flattening of the interventricular septum in systole, and the size and relative (lack of) expansion of the main and branch PAs. Cardiovascular magnetic resonance also allows measurement of aortic and PA flow for calculation of indexed cardiac output and any shunt. It also enables good visualization of any anomalies that might contribute to pulmonary hypertension such as a PDA or VSD. ${ }^{40}$ Contrast-enhanced angiography by CT or CMR may be used for the identification of thromboembolic disease or aortopulmonary collateral vessels. ${ }^{41}$ The shorter acquisition time of $\mathrm{CT}$ is an advantage in patients with limited breath-holding ability.

\section{TGA postsurgery}

Specialist knowledge is needed for adequate imaging of these patients. There have been at least 3 distinct surgical approaches to TGA. Atrial switch operation (Mustard or Senning) was widely used until a decade or so ago. The reconstructed atrial pathways then need to be appropriately imaged, as does function of the systemic RV. ${ }^{42}$ In the last decade, correction by arterial switch has gained favor, as the LV then becomes the systemic ventricle.

However, the procedure, usually performed in early infancy, involves reimplantation of the coronaries in the new aortic position and reconnection of the pulmonary trunk to what was an anteriorly located aortic root. This last maneuver results in a significant incidence of main, right, or left PA stenosis, which can be assessed by CMR. A third type of operation for TGA is the Rastelli procedure, reserved for patients who also have pulmonary stenosis and a sufficiently large subarterial VSD. After the Rastelli operation, assessment of possible stenosis or incompetence of the RV-to-PA conduit, LV outflow tract flow, biventricular function, and possible residual shunt is needed. This is probably best achieved by CMR. 


\section{Congenitally corrected TGA}

This consists of discordant atrioventricular and discordant ventriculoarterial connections (Fig 4). If uncomplicated, the affected patients can occasionally remain symptom free and undiagnosed into adulthood. Associated lesions include dextrocardia, VSD, and (sub)pulmonary stenosis. The subaortic RV is prone to dysfunction and regurgitation of its tricuspid valve. ${ }^{43}$ Which ventricle is morphologically "right" and which is "left" can be determined by the presence of multiple coarse trabeculations, including the moderator band, arising from RV but not the LV side of the septum. Each atrioventricular valve and ventriculoarterial connection should be visualized by appropriately aligned views.

\section{Fontan operations for functionally single ventricle}

Fontan operations, generally performed in children with only one effective ventricle, result in a fundamental departure from normal circulatory dynamics. ${ }^{9}$ The systemic and pulmonary vascular beds are surgically connected in series with one another downstream of the single effective ventricle, thus eliminating shunting at the cost of a critically elevated systemic venous pressure that maintains flow through the lungs. Earlier procedures incorporated the right atrium between the caval veins and PAs, whereas total cavopulmonary connection, connecting inferior vena cava flow to the PAs via a lateral tunnel or extracardiac conduit, has been favored in recent years. ${ }^{9}$ Such patients may become subject to arrhythmias, thrombosis, ascites, protein-losing enteropathy, and other complications. Patency of the cavopulmonary pathways, ventricular and valvular function, and possible causes of shunting need to be investigated. Imaging by CMR needs specific expertise and is best undertaken in a specialist ACHD center.

Complex CHD—Cardiovascular magnetic resonance allows clarification of anatomy and function, including anomalous vessels, connections, shunts, stenoses, abdominal situs, and possible polysplenia. Comprehensive cardiac, mediastinal, and upper abdominal coverage using stacks of contiguous transaxial cines is recommended, as is dynamic contrastenhanced CMR angiography. Cine images should also be aligned with each inflow and outflow valve and with any shunt flow so that connections can be established. Cardiovascular connections should be described according to sequential segmental analysis. ${ }^{44,45}$

Coronary artery anomalies-The origin and proximal course of the coronary arteries can, in most cases, be visualized by CMR; but image quality depends on meticulous technique, with fat suppression, diaphragm navigator respiratory gating, and electrocardiographic gating. Suboptimal images are prone to misinterpretation, and contrastenhanced CT may be preferable. ${ }^{46,47}$

\section{Conclusions}

After transthoracic echocardiography, which remains the first-line imaging modality in ACHD, the choice of further imaging depends on the clinical questions that remain to be addressed. Besides tissue characterization, the strengths of CMR include comprehensive access and coverage, for example, by the use of a stack of transaxial cine images or by dynamic contrast-enhanced angiography, and the relatively accurate measurements of biventricular function and volume flow. These are particularly useful in the assessment and follow-up of adults after repairs of ToF, aortic coarctation, and TGA and those with Fontan operations or with operated or unoperated complex malformations. In the author's view, a dedicated CMR service should be regarded as a necessary facility of a center specializing in the care of ACHD, with CT being preferable in selected situations. Adults with more complex conditions should ideally be investigated as well as managed in a specialist ACHD 
center. To realize their full potential and to avoid pitfalls, however, echocardiography, $\mathrm{CMR}$, and $\mathrm{CT}$ of $\mathrm{ACHD}$ require appropriate training and experience.

\section{Acknowledgments}

Support is acknowledged from the British Heart Foundation and from the National Institutes of Health Research Cardiovascular Biomedical Research Unit at the Royal Brompton and Harefield NHS Foundation Trust and Imperial College.

\section{Abbreviations and Acronyms}

ACHD adult congenital heart disease

ASD atrial septal defect

CHD congenital heart disease

CMR cardiovascular magnetic resonance

CT computed tomography

LV left ventricle

PDA patent ductus arteriosus

PA pulmonary artery

PR pulmonary regurgitation

RV right ventricle

SSFP steady-state free precession

SVC superior vena cava

TGA transposition of the great arteries

ToF tetralogy of Fallot

TR tricuspid regurgitation

VSD ventricular septal defect

\section{References}

1. Hoffman JI, Kaplan S. The incidence of congenital heart disease. J Am Coll Cardiol. 2002; 39:1890-1900. [PubMed: 12084585]

2. Marelli AJ, Mackie AS, Ionescu-Ittu R, et al. Congenital heart disease in the general population: changing prevalence and age distribution. Circulation. 2007; 115:163-172. [PubMed: 17210844]

3. Perloff JK, Warnes CA. Challenges posed by adults with repaired congenital heart disease. Circulation. 2001; 103:2637-2643. [PubMed: 11382736]

4. Silversides CK, Marelli A, Beauchesne L, et al. Canadian Cardiovascular Society 2009 Consensus Conference on the management of adults with congenital heart disease: executive summary. Can J Cardiol. 2010; 26:143-150. [PubMed: 20352134]

5. Cook SC, Raman SV. Multidetector computed tomography in the adolescent and young adult with congenital heart disease. J Cardiovasc Comput Tomogr. 2008; 2:36-49. [PubMed: 19083915]

6. Gerber TC, Carr JJ, Arai AE, et al. Ionizing radiation in cardiac imaging: a science advisory from the American Heart Association Committee on Cardiac Imaging of the Council on Clinical Cardiology and Committee on Cardiovascular Imaging and Intervention of the Council on Cardiovascular Radiology and Intervention. Circulation. 2009; 119:1056-1065. [PubMed: 19188512] 
7. Bluemke DA, Achenbach S, Budoff M, et al. Noninvasive coronary artery imaging: magnetic resonance angiography and multidetector computed tomography angiography: a scientific statement from the American Heart Association Committee on Cardiovascular Imaging and Intervention of the Council on Cardiovascular Radiology and Intervention, and the Councils on Clinical Cardiology and Cardiovascular Disease in the Young. Circulation. 2008; 118:586-606. [PubMed: 18586979]

8. Warnes CA. Transposition of the great arteries. Circulation. 2006; 114:2699-2709. [PubMed: 17159076]

9. Gewillig M. The Fontan circulation. Heart. 2005; 91:839-846. [PubMed: 15894794]

10. Kilner PJ, Geva T, Kaemmerer H, et al. Recommendations for cardiovascular magnetic resonance in adults with congenital heart disease from the respective working groups of the European Society of Cardiology. Eur Heart J. 2010; 31:794-805. [PubMed: 20067914]

11. Markl M, Kilner P, Ebbers T. Comprehensive 4D velocity mapping of the heart and great vessels by cardiovascular magnetic resonance. J Cardiovasc Magn Reson. 2011; 13:7. [PubMed: 21235751]

12. Warnes CA. Adult congenital heart disease importance of the right ventricle. J Am Coll Cardiol. 2009; 54:1903-1910. [PubMed: 19909869]

13. Piaw CS, Kiam OT, Rapaee A, et al. Use of non-invasive phase contrast magnetic resonance imaging for estimation of atrial septal defect size and morphology: a comparison with transesophageal echo. Cardiovasc Intervent Radiol. 2006; 29:230-234. [PubMed: 16252078]

14. Teo KSL, Disney PJ, Dundon BK, et al. Assessment of atrial septal defects in adults comparing cardiovascular magnetic resonance with transoesophageal echocardiography. J Cardiovasc Magn Reson. 2010; 12:44. [PubMed: 20663157]

15. Valente AM, Sena L, Powell AJ, et al. Cardiac magnetic resonance imaging evaluation of sinus venosus defects: comparison to surgical findings. Pediatr Cardiol. 2007; 28:51-56. [PubMed: 17318709]

16. Devos DG, Kilner PJ. Calculations of cardiovascular shunts and regurgitation using magnetic resonance ventricular volume and aortic and pulmonary flow measurements. Eur Radiol. 2010; 20:410-421. [PubMed: 19727751]

17. Nielsen JC, Powell AJ, Gauvreau K, et al. Magnetic resonance imaging predictors of coarctation severity. Circulation. 2005; 111:622-628. [PubMed: 15699283]

18. Therrien J, Thorne SA, Wright A, et al. Repaired coarctation: a "cost-effective" approach to identify complications in adults. J Am Coll Cardiol. 2000; 35:997-1002. [PubMed: 10732900]

19. Chessa M, Carrozza M, Butera G, et al. Results and mid-long-term follow-up of stent implantation for native and recurrent coarctation of the aorta. Eur Heart J. 2005; 26:2728-2732. [PubMed: 16186136]

20. Connolly HM, Huston J III, Brown RD Jr, et al. Intracranial aneurysms in patients with coarctation of the aorta: a prospective magnetic resonance angiographic study of 100 patients. Mayo Clin Proc. 2003; 78:1491-1499. [PubMed: 14661678]

21. Kaemmerer H, Oechslin E, Seidel H, et al. Marfan syndrome: what internists and pediatric or adult cardiologists need to know. Expert Rev Cardiovasc Ther. 2005; 3:891-909. [PubMed: 16181034]

22. Knirsch W, Kurtz C, Häffner N, et al. Dural ectasia in children with Marfan syndrome: a prospective, multicenter, patient-control study. Am J Med Genet A. 2006; 140:775-781. [PubMed: 16523504]

23. Bonow RO, Carabello BA, Kanu C, et al. ACC/AHA guidelines for the management of patients with valvular heart disease. Circulation. 2006; 114:e84-e231. [PubMed: 16880336]

24. Baumgartner H, Hung J, Bermejo J, et al. Echocardiographic assessment of valve stenosis: EAE/ ASE recommendations for clinical practice. Eur J Echocardiogr. 2009; 10:1-25. [PubMed: 19065003]

25. Gabriel RS, Kerr AJ, Raffel OC, et al. Mapping of mitral regurgitant defects by cardiovascular magnetic resonance in moderate or severe mitral regurgitation secondary to mitral valve prolapse. J Cardiovasc Magn Reson. 2008; 10:16. [PubMed: 18400088]

26. Chan KM, Wage R, Symmonds K, et al. Towards comprehensive assessment of mitral regurgitation using cardiovascular magnetic resonance. J Cardiovasc Magn Reson. 2008; 10:61. [PubMed: 19102740] 
27. Apitz C, Webb GD, Redington AN. Tetralogy of Fallot. Lancet. 2009; 374:1462-1471. [PubMed: 19683809]

28. Davlouros PA, Kilner PJ, Hornung TS, et al. Right ventricular function in adults with repaired tetralogy of Fallot assessed with cardiovascular magnetic resonance imaging: detrimental role of right ventricular outflow aneurysms or akinesia and adverse right-to-left ventricular interaction. $\mathrm{J}$ Am Coll Cardiol. 2002; 40:2044-2052. [PubMed: 12475468]

29. Geva T. Repaired tetralogy of Fallot: the roles of cardiovascular magnetic resonance in evaluating pathophysiology and for pulmonary valve replacement decision support. J Cardiovasc Magn Reson. 2011; 13:9. [PubMed: 21251297]

30. Samyn MM, Powell AJ, Garg R, et al. Range of ventricular dimensions and function by steadystate free precession cine MRI in repaired tetralogy of Fallot: right ventricular outflow tract patch vs. conduit repair. J Magn Reson Imaging. 2007; 26:934-940. [PubMed: 17896382]

31. Henkens IR, van Straten A, Schalij MJ, et al. Predicting outcome of pulmonary valve replacement in adult tetralogy of Fallot patients. Ann Thorac Surg. 2007; 83:907-911. [PubMed: 17307432]

32. Harrild DM, Berul CI, Cecchin F, et al. Pulmonary valve replacement in tetralogy of Fallot: impact on survival and ventricular tachycardia. Circulation. 2009; 119:445-451. [PubMed: 19139389]

33. Oosterhof T, van Straten A, Vliegen HW, et al. Preoperative thresholds for pulmonary valve replacement in patients with corrected tetralogy of Fallot using cardiovascular magnetic resonance. Circulation. 2007; 116:545-551. [PubMed: 17620511]

34. Lurz P, Coats L, Khambadkone S, et al. Percutaneous pulmonary valve implantation: impact of evolving technology and learning curve on clinical outcome. Circulation. 2008; 117:1964-1972. [PubMed: 18391109]

35. Frigiola A, Tsang V, Nordmeyer J, et al. Current approaches to pulmonary regurgitation. Eur J Cardiothorac Surg. 2008; 34:576-580. [PubMed: 18539471]

36. Kilner PJ, Balossino R, Dubini G, et al. Pulmonary regurgitation: the effects of varying pulmonary artery compliance, and of increased resistance proximal or distal to the compliance. Int J Cardiol. 2009; 133:157-166. [PubMed: 18722025]

37. Chaturvedi RR, Redington AN. Pulmonary regurgitation in congenital heart disease. Heart. 2007; 93:880-889. [PubMed: 17569817]

38. Kilner PJ, Sievers B, Meyer GP, et al. Double-chambered right ventricle or sub-infundibular stenosis assessed by cardiovascular magnetic resonance. J Cardiovasc Magn Reson. 2002; 4:373379. [PubMed: 12234109]

39. Geva T, Greil GF, Marshall AC, et al. Gadolinium-enhanced 3-dimensional magnetic resonance angiography of pulmonary blood supply in patients with complex pulmonary stenosis or atresia: comparison with x-ray angiography. Circulation. 2002; 106:473-478. [PubMed: 12135948]

40. Nagendran J, Michelakis E. MRI: one-stop shop for the comprehensive assessment of pulmonary arterial hypertension? Chest. 2007; 132:2-5. [PubMed: 17625076]

41. Coulden R. State-of-the-art imaging techniques in chronic thromboembolic pulmonary hypertension. Proc Am Thorac Soc. 2006; 3:577-583. [PubMed: 16963537]

42. Salehian O, Schwerzmann M, Merchant N, et al. Assessment of systemic right ventricular function in patients with transposition of the great arteries using the myocardial performance index: comparison with cardiac magnetic resonance imaging. Circulation. 2004; 110:3229-3233. [PubMed: 15533860]

43. Rutledge JM, Nihill MR, Fraser CD, et al. Outcome of 121 patients with congenitally corrected transposition of the great arteries. Pediatr Cardiol. 2002; 23:137-145. [PubMed: 11889523]

44. Anderson RH, Becker AE, Freedom RM, et al. Sequential segmental analysis of congenital heart disease. Pediatr Cardiol. 1984; 5:281-287. [PubMed: 6533609]

45. Van Praagh R, Santini F, Geva T. Segmental situs in congenital heart disease: a fundamental concept. G Ital Cardiol. 1990; 20:246-253. [PubMed: 2188858]

46. Sundaram B, Kreml R, Patel S. Imaging of coronary artery anomalies. Radiol Clin North Am. 2010; 48:711-727. [PubMed: 20705168]

47. Cheng Z, Wang X, Duan Y, et al. Detection of coronary artery anomalies by dual-source CT coronary angiography. Clin Radiol. 2010; 65:815-822. [PubMed: 20797468] 


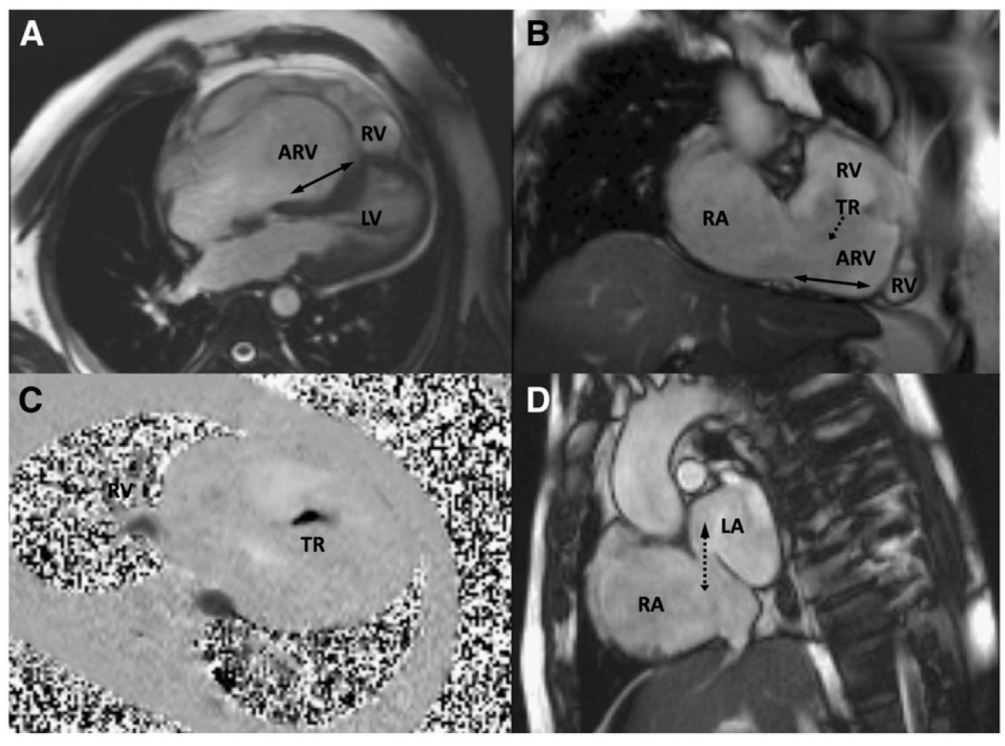

Fig 1.

Features of Ebstein anomaly in an unoperated 43-year-old patient shown by CMR. A and B, Four-chamber and oblique coronal cines show the atrialized part of the RV relative its functional part (RV). The solid double arrows indicate the extent of apical displacement of the septal and inferior insertions of the tricuspid valve. The dotted arrow indicates the direction of TR. C, Flow (dark) through a velocity mapping plane located to transect the jet of severe TR; dimensions are about $5 \times 14 \mathrm{~mm}$ in this case. D, An atrial short-axis cine shows an ostium secundum ASD (dotted double arrow) with bidirectional flow, mainly from right atrium to left atrium, in this patient. Abbreviations: ARV, atrialized part of the right ventricle; RA, right atrium; LA, left atrium. 

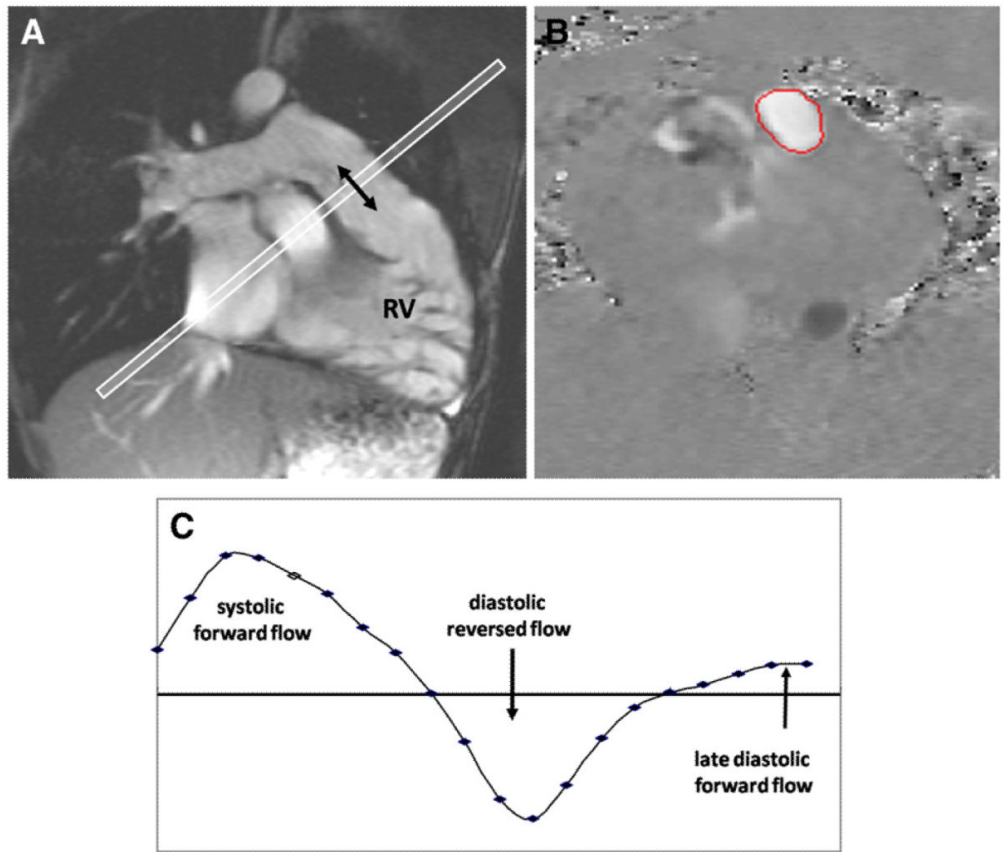

Fig 2.

Pulmonary regurgitation measured by CMR through-plane velocity mapping in repaired ToF. A, Cine imaging aligned with the RV outflow tract showed no effective pulmonary valve. B, Mapping of velocities through a plane transecting the main pulmonary artery $(\mathrm{C})$ showed systolic forward flow, diastolic reversed flow, and late diastolic forward flow at the time of atrial systole. 

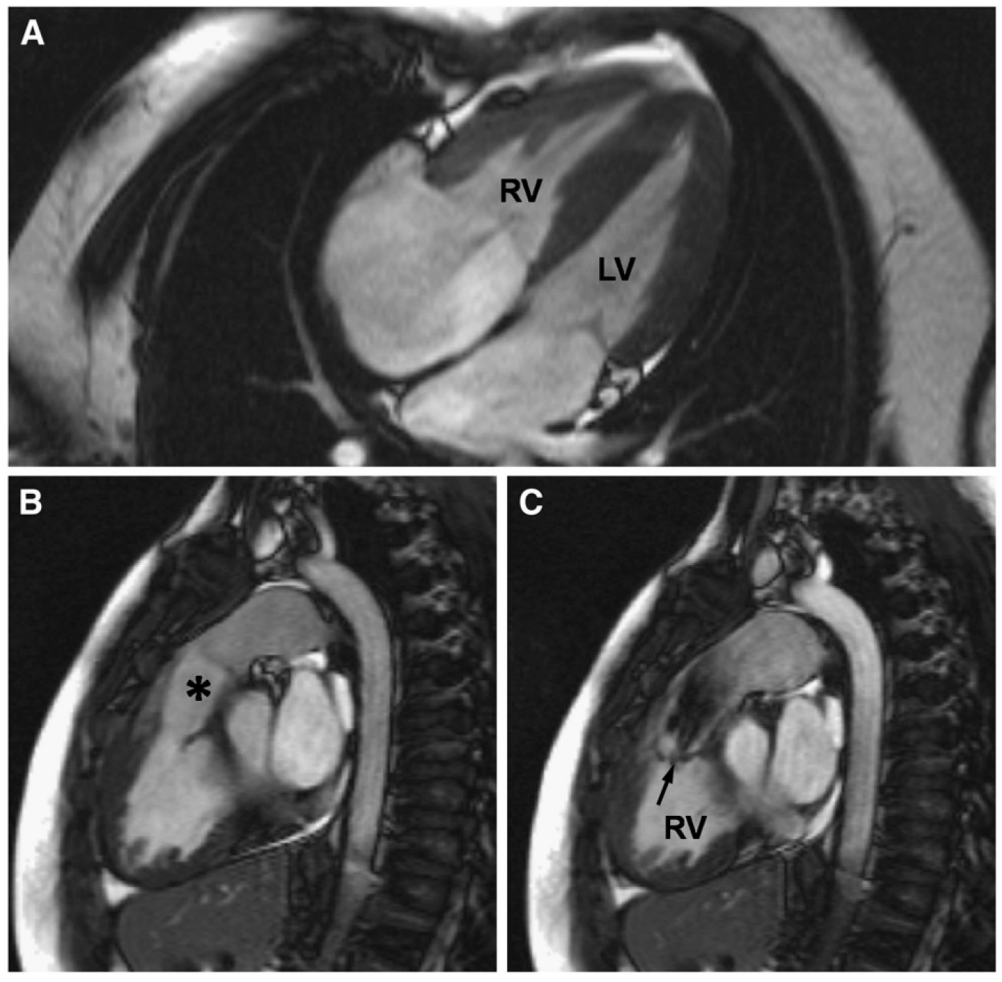

Fig 3.

Features of double-chambered RV, or subinfundibular stenosis, in an unoperated 33-yearold patient shown by CMR. A, The RV appears hypertrophied in a 4-chamber view. B, However, the infundibular region (*) and the pulmonary valve above it are unobstructed. C, The level of obstruction is seen in a systolic short-axis image. The jet from the hypertrophied part of the RV to the infundibular cavity is arrowed. 

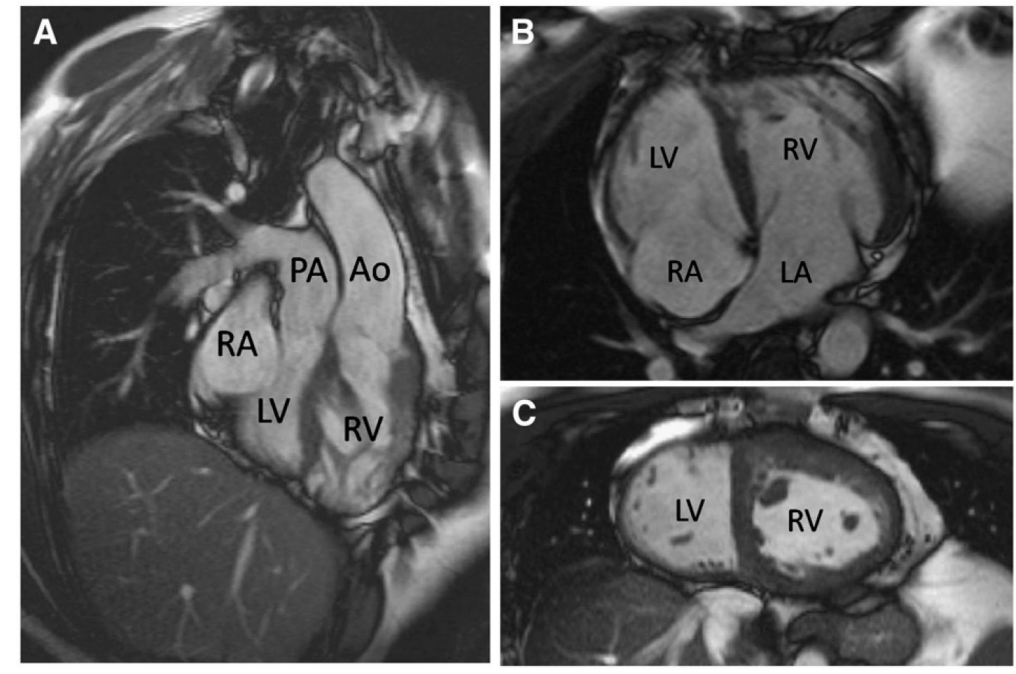

Fig 4.

Unoperated "congenitally corrected" TGA shown by CMR. Both the atrioventricular and the ventriculoarterial connections are discordant (A and B). Note the expected apical displacement of the septal insertion of the tricuspid valve of the RV relative to that of the mitral valve of the LV and the hypertrophied muscle of the systemic RV. In the mid shortaxis image (C), the LV cavity can be identified as the one on the smoother, less trabeculated sides of the ventricular septum. Abbreviations: Ao, aorta; LA, left atrium; RA, right atrium. 